\title{
Assisted Contraction
}

\author{
Fabio Finelli * \\ I.A.S.F. - Sezione di Bologna, C.N.R. \\ Via Gobetti 101, 40129 Bologna, Italy
}

November 11, 2018

\begin{abstract}
We consider the dynamics of a contracting universe ruled by two minimally coupled scalar fields with general exponential potentials. This model describes string-inspired scenarios in the Einstein frame. Both background and perturbations can be solved analytically in this model. Curvature perturbations are generated with a scale invariant spectrum only for a dust-like collapse, as happens for a single field model with an exponential potential. We find the conditions for which a scale invariant spectrum for isocurvature perturbation is generated.
\end{abstract}

\section{Introduction}

String cosmology has been recently arena of new developments. The Pre-BigBang (henceforth PBB) scenario [1, 2], starting from the low energy string effective action, included a dilaton driven phase which occurs before the radiation era. The Ekpyrotic scenario [3] uses ideas from brane cosmology inspired by string theory. According to this scenario, our universe is a 4-D orbifold fixed plane in a 5-D Horava-Witten model with a bulk brane moving towards us in the extra space dimension. Both scenarios are alternative ideas to inflation, and aim to solve the horizon problem with a phase of contraction (seen in an auxiliary conformal frame for the PBB or a physical one in the 4-D effective theory for the Ekpyrotic) rather than a superluminal expansion.

It seems difficult to obtain a scale invariant spectrum for curvature perturbations in the simplest single-field realizations of both these scenarios. Curvature perturbations have a white noise spectrum (up to a logarithmic factor) in the PBB case during the dilaton driven phase 4 , 5], and a vacuum spectrum in the Ekpyrotic scenario during the brane approach [6, 7, 8, 9]. It has been proposed for this purpose that curvature perturbations could inherit a scale invariant

*e-mail: finelli@tesre.bo.cnr.it 
spectrum from the growing mode of the Newtonian potential during the contracting phase 10] Unusual matching conditions have been proposed to this purpose 10, 11], but the debate on the spectrum of gravitational fluctuations after a bounce is still far from settled [12].

In this paper we study isocurvature perturbations arising in multifield realizations of these string-inspired cosmological models. Indeed, in the PPB scanario, a scale invariant spectrum was obtained for axion fluctuations [13]. We consider a toy model with two minimally coupled scalar field $\varphi$ and $\chi$ with generic exponential potential, described by the action:

$$
S=\int d^{4} x \sqrt{-g}\left[\frac{R}{2 \kappa^{2}}-\frac{1}{2} \partial_{\mu} \varphi \partial^{\mu} \varphi-\frac{1}{2} \partial_{\mu} \chi \partial^{\mu} \chi-V(\varphi, \chi)\right]
$$

where $\kappa^{2}=8 \pi G \equiv M_{\mathrm{pl}}^{-2}\left(M_{\mathrm{pl}}\right.$ is the reduced Planck mass $)$ and the potential is:

$$
V(\varphi, \chi)=V_{1} e^{-\beta \kappa \varphi-\lambda \kappa \chi}+V_{2} e^{-\alpha \kappa \varphi-\gamma \kappa \chi}
$$

Models of this type were studied by Liddle, Mazumdar and Schunck [14] in inflationary context and dubbed assisted inflation (see also [15, 16]). The idea is that, in the context of exponential potentials 17, several fields may cooperate to support an inflationary phase capable to produce density perturbations with an acceptable spectrum, even if each field would have a potential too steep to make it.

Exponential potentials similar to (2) are generated in several particle physics models, as in Kaluza-Klein theories, in 11-D supergravity models compactified on squashed seven spheres [18], or in generalized Scherk-Schwarz compactification of higher-dimensional Einstein vacuum space-times [19].

Here instead we study the same action (1), but leading to a RobertsonWalker metric

$$
d s^{2}=g_{\mu \nu} d x^{\mu} d x^{\nu}=-d t^{2}+a^{2}(t) d \mathbf{x}^{2}
$$

in which the scale factor $a(t)$ slowly contracts:

$$
a(t) \propto(-t)^{p}, \quad t<0, \quad 0<p<1
$$

As we have already said, these contracting space-times are of interest in string cosmology. In the Pre Big Bang scenario this contraction corresponds to a super-inflationary phase in the string frame which precedes the Big Bang. In the recently proposed Ekpyrotic scenario [3], this phase corresponds to a 4-D effective description of the approaching of the bulk brane to the orbifold we live on.

The 4-D effective action of the Ekpyrotic model includes three fields, two of which are set to be constant in the original single field model proposed in [3].

\footnotetext{
${ }^{1}$ According to this idea, the $\mathrm{PBB}$ model would have a very infrared spectrum for the Newtonian potential, as $k^{-4}$ [11].
} 
A simple form for the kinetic part of the 4-D effective action is 20:

$$
S_{k i n}=\int d^{4} x \sqrt{-g}\left[-\frac{1}{4} \partial_{\mu} \phi \partial^{\mu} \phi-\frac{3}{4} \partial_{\mu} \beta \partial^{\mu} \beta-\frac{c}{2} e^{\frac{\beta-\phi}{M_{\mathrm{pl}}}} \partial_{\mu} z \partial^{\mu} z\right],
$$

where $\phi$ is the dilaton, $\beta$ is the volume modulus and $z$ the brane modulus [20]. Once $\phi$ and $\beta$ are canonically normalized, and $z$ is kept fixed, the kinetic part of the actions (1) and (5) are the same. The kinetic part of the action (11) may also correspond to the case in which there is not a third brane in the bulk, but the boundary brane is falling on our visible brane.

According to the PBB scenario, moduli in addition to the dilaton in the Einstein frame may correspond to radii of the extra dimensions 13, 21. The action (1) would correspond to a modified scenario where both the dilaton and the moduli have exponential potentials.

The case in which the dilaton or the modulus in the effective theory of the Ekpyrotic model (5) are stabilized, leads to a two field background in which the modulus $z$ does not have a canonical kinetic term. A similar action is obtained in the PBB scenario, by considering the axion in addition to the dilaton in four dimensions. In the case of vanishing potential for $z$ or for the axion, and exponential potential for the other field, a new exact solution has been found (see second reference in [7]). Adiabatic and isocurvature perturbations in this model have been investigated only recently [22, 23].

The paper is organized as follows. In Section 2 the solution for the background evolution is presented and in Section 3 the action considered is simplified by a rotation in field space. In Section 4 adiabatic and isocurvature perturbations are studied, following the approach of Gordon et al. 24. Finally, in Section 5 we conclude.

\section{Two Field Background}

The exact solution occurs for a power-law evolution of the scale factor:

$$
a(\eta)=\left(-M_{\mathrm{pl}}(1-p) \eta\right)^{\frac{p}{1-p}},
$$

where we are restricting ourselves to the contracting case $-\infty<\eta<0$ and to $0<p<1$. The following relation exists among the exponents:

$$
\frac{p}{2}=\frac{(\beta-\alpha)^{2}+(\gamma-\lambda)^{2}}{(\beta \gamma-\lambda \alpha)^{2}} .
$$

The solution for the scalar fields are of logarithmic type (we are neglecting integration constants):

$$
\begin{aligned}
& \varphi(\eta)=A \log \left[-M_{\mathrm{pl}}(1-p) \eta\right] \\
& \chi(\eta)=C \log \left[-M_{\mathrm{pl}}(1-p) \eta\right] .
\end{aligned}
$$

\footnotetext{
${ }^{2}$ However, all the formula are valid also in the case of accellerated expansion $p>1$.
} 
The coefficients $A, C$ are related to the exponents in the following way:

$$
\begin{aligned}
\frac{A}{M_{\mathrm{pl}}} & =\frac{2}{1-p} \frac{\gamma-\lambda}{\gamma \beta-\alpha \lambda} \\
\frac{C}{M_{\mathrm{pl}}} & =\frac{2}{1-p} \frac{\beta-\alpha}{\gamma \beta-\alpha \lambda}
\end{aligned}
$$

and

$$
\frac{V_{1}+V_{2}}{M_{\mathrm{pl}}^{4}}=p(3 p-1)
$$

We now introduce the average field $\sigma$ and its orthogonal field $s$, by following the formalism of Ref. 24] P. This method will be particularly useful for the study of adiabatic and isocurvature perturbations in the next section. The fields $\sigma$ and $s$ are obtained by a rotation of $\varphi$ and $\sigma$ :

$$
\begin{array}{r}
\mathrm{d} \sigma=\cos \theta \mathrm{d} \varphi+\sin \theta \mathrm{d} \chi \\
\mathrm{d} s=-\sin \theta \mathrm{d} \varphi+\cos \theta \mathrm{d} \chi
\end{array}
$$

where

$$
\begin{aligned}
& \cos \theta=\frac{\dot{\varphi}}{\sqrt{\dot{\varphi}^{2}+\dot{\chi}^{2}}}=\frac{\dot{\varphi}}{\dot{\sigma}}=\frac{A}{M_{\mathrm{pl}}} \frac{1-p}{\sqrt{2 p}} \\
& \sin \theta=\frac{\dot{\chi}}{\sqrt{\dot{\varphi}^{2}+\dot{\chi}^{2}}}=\frac{\dot{\chi}}{\dot{\sigma}}=\frac{C}{M_{\mathrm{pl}}} \frac{1-p}{\sqrt{2 p}} .
\end{aligned}
$$

Since both $\varphi$ and $\chi$ scale in the same (logarithmic) way, $\theta$ is constant:

$$
\theta=\arctan \frac{C}{A} \text {. }
$$

This means that the motion occurs in a straight line in the field space $(\varphi, \chi)$.

\section{Rotation in Field Space}

It is interesting to rewrite the action (1) in terms of the average field $\sigma$ and the orthogonal field $s$ [15]. This procedure is particularly easy for exponential potentials, since it is possible to integrate directly Eqs. (12), being $\theta$ constant in time. Therefore the action in Eq. (11) with the rotated fields is:

$$
S=\int d^{4} x \sqrt{-g}\left[\frac{R}{2 \kappa^{2}}-\frac{1}{2} \partial_{\mu} \sigma \partial^{\mu} \sigma-\frac{1}{2} \partial_{\mu} s \partial^{\mu} s-e^{-\sqrt{\frac{2}{p}} \kappa \sigma} \bar{V}(s)\right]
$$

\footnotetext{
${ }^{3}$ See also the same formalism used as a rotation in the background field space in the context of assisted inflation 15. We note that this rotation always leads to a second field $s$, orthogonal to $\sigma$, which remains constant in time by definition.
} 


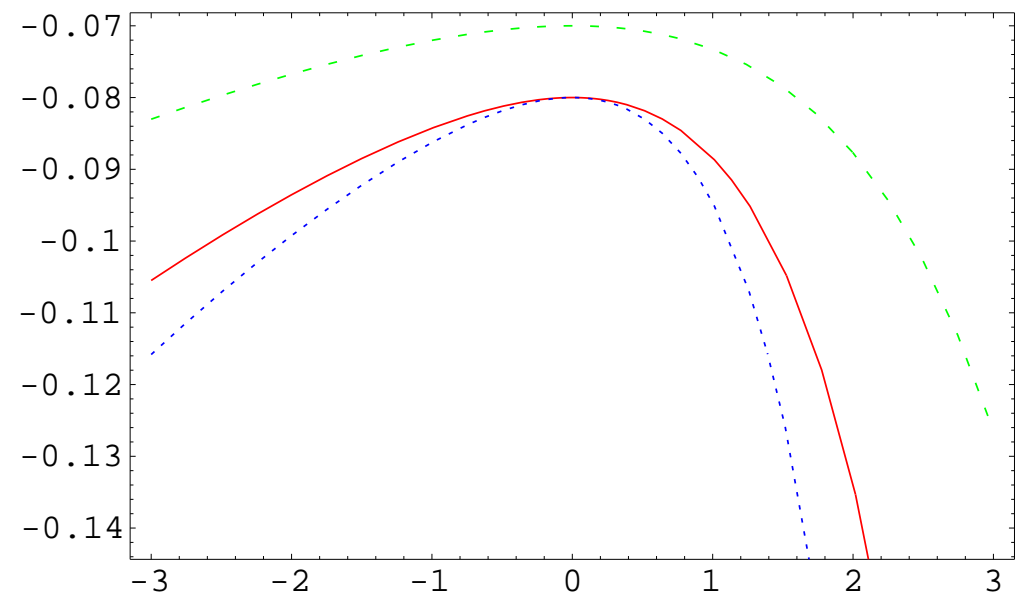

Figure 1: The potential $\bar{V}(s)$ (in units of $M_{\mathrm{pl}}^{4}$ ) as a function of $s / M_{\mathrm{pl}}$ for $p=0.2$, $d=4$ (solid line) and $d=5$ (dotted line), and for $p=0.1, d=4$ (dashed line). For all the curves $x=0.1$.

with

$$
\begin{aligned}
\bar{V}(s) & =V_{1} e^{-\kappa s(\lambda \cos \theta-\beta \sin \theta)}+V_{2} e^{-\kappa s(\gamma \cos \theta-\alpha \sin \theta)} \\
& =M_{\mathrm{pl}}^{4} p(3 p-1)\left[x e^{\sqrt{p / 2} d \kappa s(1-x)}+(1-x) e^{-\sqrt{p / 2} d \kappa s x}\right],
\end{aligned}
$$

where we have neglected the integration constants in integrating Eq. (12) (which would just modify $V_{1}, V_{2}$ ), we have defined $d=\gamma \beta-\alpha \lambda$ and introduced

$$
x=\frac{V_{1}}{p(3 p-1) M_{\mathrm{pl}}^{4}} .
$$

By definition $s$ is constant, which means that $s$ sits in the extremum of the potential (16), $s_{\min }=0$. In Fig. 1 we show the potential $\bar{V}(s)$ for $x=0.1$ and its dependence on $p$ and $d$. It is instructive to study the potential $\bar{V}(s)$ close to the extremum, by Taylor expanding:

$$
\bar{V}(s)=M_{\mathrm{pl}}^{4} p(3 p-1)\left\{1+\frac{s^{2}}{2 M_{\mathrm{pl}}^{2}} x(1-x)\left[(\beta-\alpha)^{2}+(\gamma-\lambda)^{2}\right]+\mathcal{O}\left(s^{3}\right)\right\} .
$$

For a better understanding of the form of the potential in (15), it is also possible to write the problem in a string frame conformally related to the metric $g_{\mu \nu}$ used in (3). By following Ref. [15], we get (by neglecting boundary terms):

$$
\tilde{S}=\int d^{4} x \sqrt{-\tilde{g}} e^{-\tilde{\sigma}}\left[\frac{1}{2 \kappa^{2}}\left(\tilde{R}-\omega \tilde{g}^{\mu \nu} \partial_{\mu} \tilde{\sigma} \partial_{\nu} \tilde{\sigma}\right)-\frac{1}{2} \tilde{g}^{\mu \nu} \partial_{\mu} s \partial_{\nu} s-\bar{V}(s)\right],
$$


where $\tilde{\sigma}=-\sqrt{2 / p} \kappa \sigma$ and

$$
\tilde{g}_{\mu \nu}=e^{\tilde{\sigma}} g_{\mu \nu} \quad \omega=\frac{p-3}{2} .
$$

We note that for $p=1$ the kinetic term of $\tilde{\sigma}$ is just the usual one considered in the low-effective action for string theory in four dimension.

\section{Perturbations}

We now consider the perturbations around the background defined in the previous section. We shall focus directly on adiabatic and isocurvature fluctuations. We use the curvature perturbation $\zeta$ 25, 26] to follow the evolution of the adiabatic mode. In the longitudinal gauge for metric perturbations:

$$
d s^{2}=-(1+2 \Phi(t, \mathbf{x})) d t^{2}+a(t)^{2}(1-2 \Phi(t, \mathbf{x})) d \mathbf{x}^{2}
$$

the variable $\zeta$ is 26:

$$
\zeta=-\frac{H}{\dot{H}}(\dot{\Phi}+H \Phi)+\Phi=\frac{H}{\dot{\sigma}} \delta \sigma+\Phi,
$$

where in last equality $\zeta$ is expressed as function of the fluctuation of the average field [24]. The time evolution of $\zeta$ is [27, 28]:

$$
\dot{\zeta}=-\frac{H}{\dot{H}} \frac{\nabla^{2}}{a^{2}} \Phi+\frac{H}{2}\left(\frac{\delta \varphi}{\dot{\varphi}}-\frac{\delta \chi}{\dot{\chi}}\right) \frac{d}{d t}\left(\frac{\dot{\varphi}^{2}-\dot{\chi}^{2}}{\dot{\varphi}^{2}+\dot{\chi}^{2}}\right)=-\frac{H}{\dot{H}} \frac{\nabla^{2}}{a^{2}} \Phi+\frac{2 H}{\dot{\sigma}} \dot{\theta} \delta s
$$

As follows from Ref. 24 curvature and isocurvature perturbations are decoupled when $\dot{\theta}=0$. Therefore, in the case of exponential potentials curvature $\zeta$ and isocurvature $\delta s$ perturbations are a particular rotation in the space of perturbations which diagonalize the system of equations of motion. The equation for the Fourier mode of $\zeta$ is:

$$
\ddot{\zeta}_{k}+\left(3 H-2 \frac{\dot{H}}{H}+\frac{\ddot{H}}{\dot{H}}\right) \dot{\zeta}_{k}+\frac{k^{2}}{a^{2}} \zeta_{k}=0
$$

For power-law evolution of the scale factor as in Eq. (6), the damping term is simply $3 H \dot{\zeta}$ and therefore Eq. (24) is the equation for a massless minimally coupled scalar field. In the single field case $\zeta$ satisfies the same equation [6]. For an evolution of the scale factor given by Eq. (6), the solution for $\zeta$ is [7]:

$$
\zeta_{k}=\frac{1}{2 a M_{\mathrm{pl}}} \sqrt{-\frac{\pi p \eta}{2}} H_{\left|\nu_{\zeta}\right|}^{(2)}(-k \eta),
$$

where $H_{|\nu|}^{(2)}$ is the Hankel function of the second kind and the spectral index $\nu$ is given:

$$
\nu_{\zeta}=\frac{1}{2} \frac{1-3 p}{1-p} .
$$


A scale invariant spectrum results only for a dust-like collapse, i.e. $p=2 / 3$ [7]. For an extremely slow contraction, i.e. $p \sim 0$, as suggested in the Ekpyrotic scenario [3], $\zeta$ has a blue spectrum [6, 7, 8, 9].

We now study the solution for the isocurvature perturbations. The equation for $\delta s_{k}$ with $\dot{\theta}=0$ can be rewritten as [24]:

$$
\left(a \delta s_{k}\right)^{\prime \prime}+\left[k^{2}+a^{2} V_{s s}-\frac{a^{\prime \prime}}{a}\right]\left(a \delta s_{k}\right)=0 .
$$

where

$$
\begin{gathered}
V_{s s}=\sin ^{2} \theta V_{\varphi \varphi}-\sin 2 \theta V_{\varphi \chi}+\cos ^{2} \theta V_{\chi \chi} \\
\frac{a^{\prime \prime}}{a}=p \frac{2 p-1}{(1-p)^{2} \eta^{2}} .
\end{gathered}
$$

The solution for $\delta s_{k}$ is:

$$
a \delta s_{k}=\left(-\frac{\pi \eta}{4}\right)^{1 / 2} H_{\left|\nu_{s}\right|}(-k \eta)
$$

where $\nu_{s}$ can be obtained from:

$$
\frac{\nu_{s}^{2}-1 / 4}{\eta^{2}}=\frac{a^{\prime \prime}}{a}-a^{2} V_{s s} .
$$

By inserting Eqs. (6-13) in Eq. (28) one gets:

$$
a^{2} V_{s s}=\frac{V_{1} V_{2}}{M_{\mathrm{pl}}^{8}(3 p-1)} \frac{d^{2}}{2(1-p)^{2} \eta^{2}}
$$

By using Eq. (11), it is useful to rewrite this last equation as:

$$
\begin{aligned}
a^{2} V_{s s} & =p^{2} d^{2} x(1-x) \frac{3 p-1}{2(1-p)^{2} \eta^{2}} \\
& =p\left[(\beta-\alpha)^{2}+(\gamma-\lambda)^{2}\right] x(1-x) \frac{3 p-1}{(1-p)^{2} \eta^{2}}
\end{aligned}
$$

The effective mass for the isocurvature perturbations is therefore zero when $V_{1}$ or $V_{2}$ vanish. This means that for a potential which is just a product of exponentials of the single fields, adiabatic and isocurvature fluctuations are decoupled, but generated with the same spectrum, produced by the curvature term $a^{\prime \prime} / a$. The same thing occurs for a free theory $\left(V_{1}=V_{2}=0, p=1 / 3\right)$, a result which is already known in the PBB scenario [13]. We also note that the effective mass in Eq. (33) agrees with the curvature of the potential in Eq. (18). For $0<x<1$ the potential $V$ around $s=0$ is concave (convex) for $0<p<1 / 3$ $(1 / 3<p<1)$. 
We now consider the case for which the spectrum of isocurvature perturbations is scale invariant, i.e. when the following condition is satisfied:

$$
a^{2} V_{s s}-\frac{a^{\prime \prime}}{a}=-\frac{2}{\eta^{2}} .
$$

By solving the second order equation for $x$ deriving from the above equation we find:

$$
x_{1,2}=\frac{1}{2} \pm \sqrt{\frac{1}{4}+\frac{4}{p^{2} d^{2}(3 p-1)}\left(1-\frac{3}{2} p\right)} .
$$

In what follows we shall discuss some particular cases.

CASE A: $|\gamma|,|\beta| \gg|\lambda|,|\alpha|$.

It is useful to rewrite Eqs. (10) with the help of Eqs. (13):

$$
\begin{aligned}
& \gamma=\lambda+\sqrt{\frac{p}{2}}(\gamma \beta-\alpha \lambda) \cos \theta \\
& \beta=\alpha+\sqrt{\frac{p}{2}}(\gamma \beta-\alpha \lambda) \sin \theta
\end{aligned}
$$

We now work in the regime in which $|\gamma|,|\beta| \gg|\lambda|,|\alpha|$, which leads to:

$$
\begin{aligned}
& \beta \sim \sqrt{\frac{2}{p}} \frac{1}{\cos \theta} \\
& \gamma \sim \sqrt{\frac{2}{p}} \frac{1}{\sin \theta} .
\end{aligned}
$$

If also $p \sim 0$ we have $|\gamma|,|\beta| \gg 1$. Under these two conditions it is easy to show that $a^{2} V_{s s} \sim-2 / \eta^{2}$ and therefore

$$
\nu_{s}^{2}=\frac{9}{4}-\sqrt{\frac{p}{2}}\left(\frac{\alpha}{\cos \theta}+\frac{\lambda}{\sin \theta}\right)+\mathcal{O}(p),
$$

i.e. the spectrum of isocurvature perturbations is nearly scale invariant, with a tilt of the order of $\sqrt{p}$.

By symmetry, an analog result holds in the opposite case, i. e. $|\gamma|,|\beta| \ll$ $|\lambda|,|\alpha|$, when $p \sim 0$. In this case the spectral index is the same of Eq. (38), with $\alpha$ and $\lambda$ replaced with $\beta$ and $\gamma$, respectively.

CASE B: $\lambda=\alpha=0$. 


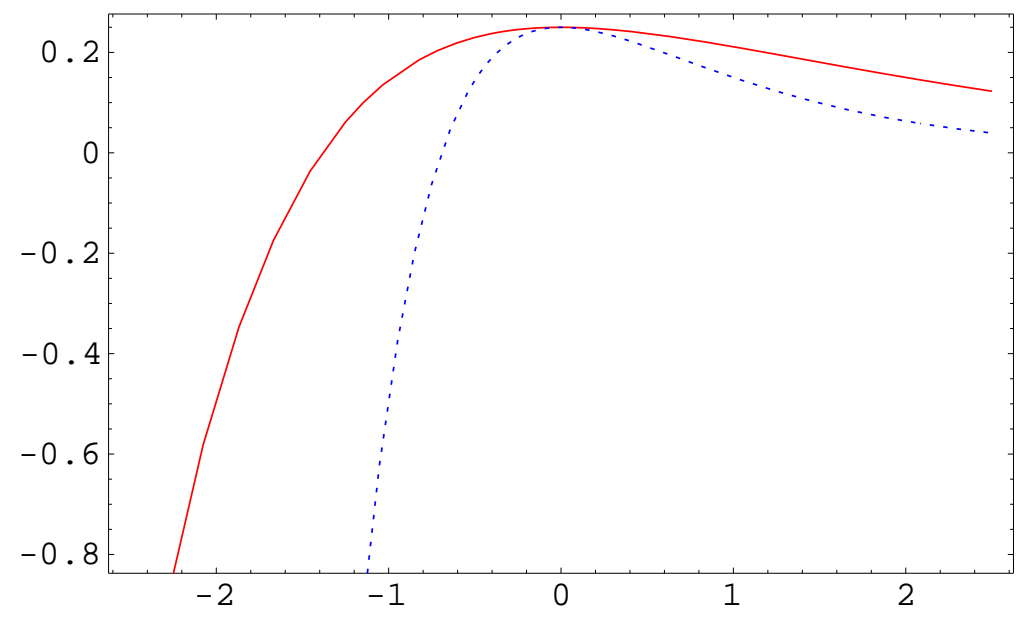

Figure 2: The potential $\bar{V}(s)$ (in units of $M_{\mathrm{pl}}^{4}$ ) as a function of $s / M_{\mathrm{pl}}$ for $p=1 / 2$ case, with $d=1$ (solid line) and $d=2$ (dotted line). For both curves $x=2$.

In this case we get the following result for the spectral index in Eq. (31):

$$
\nu_{s}=\frac{3}{2} \frac{\sqrt{(1 / 3-p)(3-p)}}{1-p} .
$$

The same result was found in [15]. We note that the spectral index does not depend on $\theta$, i.e. on how the total energy is shared between the two fields. For $1 / 3<p<1, \nu_{s}$ is imaginary, which means that isocurvature perturbations are exponentially dumped. For $0<p<1 / 3, \nu_{s}$ is real, and in the case of a very slow contraction $p \sim 0$ we get a nearly scale-invariant spectrum, slightly blue-tilted:

$$
\nu_{s}=\frac{3}{2}-p+\mathcal{O}\left(p^{2}\right) \text {. }
$$

CASE C: $p=\frac{1}{2}$.

In this case we have a radiation type contraction, in which the curvature term $a^{\prime \prime} / a$ is zero. For $p=1 / 2$ a scale invariant spectrum for isocurvature perturbations emerges only when $V_{1}$ and $V_{2}$ have opposite signs $(x<0$ or $x>1$ ). We show in Fig. (2) the shape of the potential $\bar{V}(s)$ for $x=2$ for two possible values of $d$.

The 3-D plot of $\nu_{s}^{2}$ as a function of $x$ and $d$ is presented in Fig. (3). As follows from the general case treated in Eq. (35), $\nu_{s}=3 / 2$ emerges when

$$
x_{1,2}^{p=1 / 2}=\frac{1}{2} \pm \sqrt{\frac{1}{4}+\frac{8}{d^{2}}}
$$




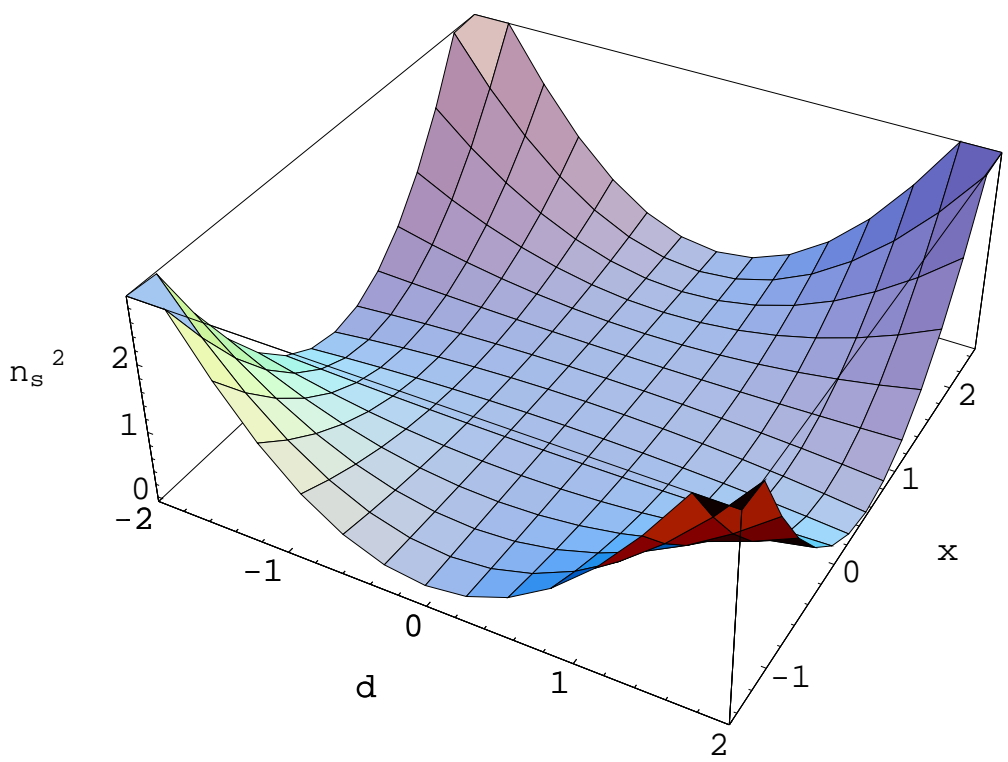

Figure 3: The spectral index for isocurvature perturbations in the case $p=1 / 2$ as a function of $d$ and $x$.

\section{Discussions and Conclusions}

We have discussed a scenario with a contracting universe driven by two minimally coupled scalar fields with a generic exponential potential. This model allows exact solutions for background and cosmological perturbations and is relevant in string cosmology [1, 2, 34, studied in the Einstein frame. The corresponding solution with an expanding universe has been considered in the framework of assisted inflation [14].

The action considered in Eq. (11) has a more appealing form by rewriting it as a function of the average and the orthogonal field, as in Eq. (15,19). A similar action was considered in the expanding case in soft inflation models [29].

As already mentioned, for the action (1,2) exact solutions are possible for adiabatic and isocurvature perturbations, since these are decoupled. In the inflationary context, adiabatic and isocurvature perturbations are nearly scale invariant for the same condition, i.e. $p>>1$ [15, as follows from Eqs. (26, 39). The reason being that both adiabatic and isocurvature perturbations are amplified by the curvature term $a^{\prime \prime} / a$ in the range $p>>1$. Instead, during a contraction, isocurvature perturbation can develop a scale invariant spectrum for $p \sim 0$, while curvature perturbation cannot. The curvature term $a^{\prime \prime} / a$ is suppressed by $p$ and ineffective for the amplification of $\zeta$, while the effective mass for isocurvature perturbation is negative, and the generation of a scale invariant spectrum for the latter is possible.

We have shown that the mechanism to produce a scale invariant spectrum

\footnotetext{
${ }^{4}$ The effective mass for isocurvature perturbations is suppressed by $p$ with respect to the curvature term $a^{\prime \prime} / a$ for $p>>1$, as it can be seen from Eqs. (17.33).
} 
based on tachyonic instability, rather than the geometric effect due to the curvature term $a^{\prime \prime} / a$, proposed in [30], could really work in a multifield scenario. This result is also attractive from the particle physics point of view, since tachyonic instability occurs in symmetry breaking phenomena, mostly implemented with two or more fields. In the single field case, the negative effective mass, originating from the negative exponential potential, is balanced by the metric contribution (indeed, gravity is ruled by this single field) and a scale invariant spectrum for curvature perturbation cannot be generated [6, 7], at least for exponential potentials. In the multifield case, this result is confirmed by the equation of motion for the adiabatic perturbation (24), which is the same of a single field one for power-law expansion. For the isocurvature perturbation $\delta s$ this relation between its effective potential and the metric contribution is lost. A negative effective mass could really survive and lead to an infrared spectrum, as shown here.

We think this isocurvature mechanism works in a generic way, and it does not occur only with the exponential potential we started from. For the potential (2), $s$ is exactly frozen, but one can guess that the result will not be very different when $s$ slowly moves, as it happens in the slow-rollover regime for the inflaton. Indeed, from Eq. (18) the potential around the extremum is very simple both in the case B:

$$
\bar{V}(s) \simeq-p M_{\mathrm{pl}}^{4}-M_{\mathrm{pl}}^{2} s^{2} .
$$

and in the case $\mathrm{C}$ :

$$
\bar{V}(s) \simeq \frac{M_{\mathrm{pl}}^{4}}{4}\left(1-\frac{s^{2}}{M_{\mathrm{pl}}^{2}}\right) .
$$

We have concentrate our attention on contractions for which the curvature $a^{\prime \prime} / a$ is minimal, i.e. $p \sim 0$ and $p \sim 1 / 2$. In our opinion, these types of contractions would alleviate (but not solve) the problem of amplifying classical inhomogeneities simultaneously with quantum fluctuations [31]. Initial curvature perturbations are almost inert because the curvature is almost zero, while isocurvature perturbations are amplified by tachyonic instability.

It remains to see how and when the scale invariant spectrum of isocurvature perturbations is transferred to the adiabatic component. For exponential potential adiabatic and isocurvature perturbations are decoupled, but for potentials like in Eqs. (42,43) are coupled (weakly if $s$ slowly rolls). Of course, a transfer could also occur during the bounce - or the graceful exit - or during a stabilization mechanism, but this possibility remains to be investigated in detail.

\section{Acknowledgements}

It is a pleasure to thank Robert Brandenberger for several important suggestions and useful comments on the manuscript, and Ruth Durrer for interesting 
discussions.

\section{References}

[1] M. Gasperini and G. Veneziano, Astropart. Phys. 1, 317 (1993)

[2] G. Veneziano, arXiv:hep-th/0002094.

[3] J. Khoury, B. A. Ovrut, P. J. Steinhardt and N. Turok, Phys. Rev. D 64, $123522(2001)$.

[4] R. Brustein, M. Gasperini, M. Giovannini, V. F. Mukhanov and G. Veneziano, Phys. Rev. D 51, 6744 (1995)

[5] N. Deruelle and V. F. Mukhanov, Phys. Rev. D 52, 5549 (1995)

[6] D. Lyth, Phys. Lett. B 524, 1 (2002); ibid. B 526, 173 (2002).

[7] R. Brandenberger and F. Finelli, JHEP 0111, 056 (2001); F. Finelli and R. Brandenberger, Phys. Rev. D 65, 103522 (2002).

[8] J. c. Hwang, Phys. Rev. D 65, 063514 (2002)

[9] S. Tsujikawa, Phys. Lett. B 526, 179 (2002).

[10] J. Khoury, B. A. Ovrut, P. J. Steinhardt and N. Turok, arXiv:hepth/0109050.

[11] R. Durrer, arXiv:hep-th/0112026; R. Durrer and F. Vernizzi, arXiv:hep$\mathrm{ph} / 0203275$.

[12] J. c. Hwang and H. Noh, arXiv:astro-ph/0112079; J. Hwang and H. Noh, arXiv:hep-th/0203193; J. Martin, P. Peter, N. Pinto Neto and D. J. Schwarz, arXiv:hep-th/0112128; J. Martin, P. Peter, N. Pinto-Neto and D. J. Schwarz, arXiv:hep-th/0204222; P. Peter, J. Martin, N. PintoNeto and D. J. Schwarz, arXiv:hep-th/0204227; P. Peter and N. Pinto-Neto, arXiv:hep-th/0203013.

[13] E. J. Copeland, R. Easther and D. Wands, Phys. Rev. D 56, 874 (1997)

[14] A. R. Liddle, A. Mazumdar, F. E. Schunck, Phys. Rev. D 58, 061301 (1998).

[15] K. Malik and D. Wands, Phys. Rev. D 59, 123501 (1999).

[16] E. J. Copeland, A. Mazumdar, and N. J. Nunes, Phys. Rev. D 60, 083506 (1999).

[17] F. Lucchin and S. Matarrese, Phys. Rev. D 32, 1316 (1985). 
[18] S. W. Hawking, H. S. Reall, Phys. Rev. D 59, 023502 (1999).

[19] A. M. Green and J. E. Lidsey, Phys. Rev. D 61, 067301 (2000).

[20] E. J. Copeland, J. Gray, and A. Lukas, Phys. Rev. D 64, 126003 (2001).

[21] A. Buonanno, K. A. Meissner, C. Ungarelli, G. Veneziano, Phys. Rev. D 57, 2543 (1998).

[22] A. Notari and A. Riotto, arXiv:hep-th/0205019.

[23] R. Brandenberger, F. Di Marco, and F. Finelli, in preparation (2002).

[24] C. Gordon, D. Wands, B. A. Bassett, and R. Maartens, Phys. Rev. D 63, 023506 (2001).

[25] D. H. Lyth, Phys. Rev. D 31, 1792 (1985).

[26] V. F. Mukhanov, H. A. Feldman and R. H. Brandenberger, Phys. Rept. 215, 203 (1992).

[27] J. Garcia-Bellido and D. Wands, Phys. Rev. D 53, 5437 (1996).

[28] F. Finelli and R. Brandenberger, Phys. Rev. D 62, 083502 (2000)

[29] A. L. Berkin, K. Maeda, and J. Yokoyama, Phys. Rev. Lett. 65, 141 (1990).

[30] R. Kallosh, L. Kofman, and A. Linde, Phys. Rev. D 64, 123523 (2001).

[31] A. D. Linde, arXiv:hep-th/0107176 and arXiv:hep-th/0205259. 